\title{
Solute Diffusion in Hydrogels and Polymer Solutions
}

\author{
Brian G. Amsden \\ Queen's University, Canada \\ 99 University Ave, Kingston, ON K7L 3N6 \\ brian.amsden@queensu.ca
}

Hydrogels are used in a wide variety of fields, including separation processes such as chromatography, the encapsulation of cells for both biomedical and fermentation purposes, in biosensors, and as biomaterials for the delivery of bioactive agents to the body as well as scaffolds for tissue engineering and regenerative medicine. Further, diffusion of small molecules, globular proteins and colloidal particles in aqueous polymer solutions is important for drug diffusion in biological fluids and in the release of drugs from formulations such as tablets. The one feature of hydrogels and polymer solutions that all these applications capitalize upon is the ability of the polymer chains in solution to restrict the diffusive movement of a solute. For design purposes it would be useful to have a mathematical expression that provides reliable estimates of solute diffusion coefficients within hydrogels. In this talk, the development and utility of a model based on obstruction effects will be presented and its ability to predict solute diffusion coefficients within hydrogels and polymer solutions demonstrated. 